Stockholm

USITP 99-4

June 1999

\title{
DE SITTER SPACE AND SPATIAL TOPOLOGY
}

\author{
Ingemar Bengtsson円 \\ Sören Holst? \\ Fysikum \\ Stockholm University \\ Box 6730, S-113 85 Stockholm, Sweden
}

\begin{abstract}
Morrow-Jones and Witt have shown that generic spatial topologies admit initial data that evolve to locally de Sitter spacetimes under Einstein's equations. We simplify their arguments, make them a little more general, and solve for the global time evolution of the wormhole initial data considered by them. Finally we give explicit examples of locally de Sitter domains of development whose universal covers cannot be embedded in de Sitter space.
\end{abstract}

\footnotetext{
${ }^{1}$ Email address: ingemar@physto.se. Supported by NFR.

${ }^{2}$ Email address: holst@physto.se
} 


\section{INTRODUCTION.}

In a paper entitled "Inflationary data for generic spatial topology" MorrowJones and Witt [1] were able to show that it is possible to set initial data on three-spaces of generic topology which evolve to locally de Sitter spacetimes under the equation

$$
R_{\alpha \beta}=\lambda g_{\alpha \beta}, \quad \lambda>0 .
$$

During inflation - if it occurs - this is indeed how the Universe evolves. Given the continuing interest in both non-trivial spatial topologies and inflation it seems worthwhile to explore these results a little further. Let us briefly summarize the main points of Morrow-Jones and Witt: According to a widely believed conjecture [2], all three-manifolds can be "glued together" from certain basic building blocks. These building blocks are Thurston's model geometries. There are altogether eight model geometries of which four are locally spherically symmetric, namely hyperbolic space $\mathbf{H}^{3}$, flat space $\mathbf{E}^{3}$, the three-sphere $\mathbf{S}^{3}$, and the handle $\mathbf{S}^{2} \times \mathbf{E}$. Naturally we must allow the topologies that can be obtained by taking the quotient of a building block with some discrete isometry group $\Gamma$. In a well defined sense "generic" spatial topologies are necessarily of the form $\mathbf{H}^{3} / \Gamma$ [2], so including further model geometries makes the topology generic by default. The first observation in ref. [1] is that all the four model geometries that we have mentioned can be embedded as spatial hypersurfaces in de Sitter space, so that locally de Sitter initial data can be set on all of them as well as on their quotients. Next the "gluing together" is done by taking a connected sum, which means that one removes a ball from each piece and identifies the resulting boundaries. While it is clear that this can be done in a way that preserves the local spherical symmetry of the three-metrics, it is not obvious that the full set of initial data for Einstein's equations can be chosen so that spacetime remains locally de Sitter. Indeed since there are elliptic constraint equations one expects the presence of a handle on a sphere (say) to affect the geometry all over the sphere, so that attaching a second handle might prove difficult. Nevertheless Morrow-Jones and Witt were able to show that such "Machian" behaviour is in fact absent provided that the minimum radius $R$ of the han-

dles is large enough. To show that the connected sums can indeed evolve to locally de Sitter spacetimes Morrow-Jones and Witt present a slightly com- 
plicated argument that relies on a cosmic Birkhoff theorem and analyticity in the various charts.

In this paper we will rederive and extend the above results in two different ways: First we rewrite the de Sitter metric by reparametrizing two of the coordinates. This brings two arbitrary functions of each coordinate into the metric, and we think of these two functions as providing the general solution of the two dimensional wave equation. Effectively, this means that we can solve the main problem of ref [1] by choosing initial data for the wave equation rather than for Einstein's equation. (This method can be applied in more general situations but turns out to be useful here only because the de Sitter metric is so simple.) Then we present a pictorial description of de Sitter space that allows us to drop the restriction of local spherical symmetry everywhere, and to see the global time evolution of the data at a glance. We use the pictorial method to address an interesting point raised by MorrowJones and Witt (and known to mathematicians [3]), namely the existence of smooth locally de Sitter domains of development whose universal covers cannot be embedded in de Sitter space. It turns out that $\mathbf{H}^{2} \times \mathbf{E}$, the fifth of Thurston's model geometries, serves as an example. (On the other hand we will find that the example proposed by Morrow-Jones and Witt does not work.)

Throughout, a "spacetime" means the domain of development of some complete smooth initial data surface. It will become clear that most of the spacetimes constructed are complete only in one time direction, chosen to be the future. If we evolve back into the past we will typically encounter either Cauchy horizons, Misner type singularities, or both. In some cases the solution can be analytically extended across the horizon, and then the spatial topology may be revealed as being in some sense not what we thought it was. An example would be an $\mathbf{H}^{3}$ initial data surface, for which the solution can be extended to ordinary de Sitter space whose topology is in fact $\mathbf{S}^{3} \times \mathbf{R}$. In other cases there are singularities in the past, perhaps making it impossible to continue the solution across the Cauchy horizon. An example would be $\mathbf{H}^{3} / \Gamma$ for a suitably chosen discrete group [4]. We choose to ignore this point, which means that we have nothing to say about the issue of how inflation started.

The organization of our paper is as follows: In section 2 we rederive the central result of ref. [1] by writing the de Sitter metric in a form where picking a locally spherically symmetric geometry on an initial data slice is 
achieved by choosing initial data for the two dimensional wave equation. In section 3 we show - using the hyperboloid model and following Schrödinger [5] - how some of Thurston's model geometries can be embedded in a de Sitter space. In section 4 we explain how de Sitter space can be visualized in intrinsic terms; from here on the argument relies to a large extent on pictures. Section 5 is devoted to some of the wormhole topologies discussed by Morrow-Jones and Witt [1]. Finally, in section 6 we construct examples of locally de Sitter spacetimes whose universal covers cannot be found as a subset of de Sitter space and in section 7 we summarize our conclusions.

\section{A USEFUL FORM OF THE DE SITTER METRIC.}

We define a locally spherically symmetric three-space as a space that can be covered with charts in which the metric takes the form

$$
d l^{2}=a^{2}\left(d r^{2}+f^{2}(r) d \Omega^{2}\right),
$$

where $d \Omega^{2}$ is the metric on the two-sphere $\mathbf{S}^{2}$ and $a$ is some constant. Examples include four of the five model geometries mentioned above as well as their connected sums [1]. The point is that the connected sum can be taken by removing a sphere symmetrically placed within a chart of this type. We then choose an $f$ that interpolates between what we need for the model geometries that are to be connected together, and we are done. The question to be investigated is therefore: What restrictions on $f$ are imposed by the requirement that such a three-space can be embedded in a locally de Sitter space?

Our strategy in this section is to reparametrize the de Sitter metric in such a way that it contains a function $F(t, r)$; the metric will remain de Sitter if and only if $F$ is a solution of the two dimensional wave equation. By inspection we will then be able to see that choosing the function $f(r)$ and the constant $a$ in the induced 3-metric at constant $t$ corresponds to choosing initial data for the wave equation. A restriction on possible forms of $f$ will arise from the demand that the 3-metric shall have Euclidean signature. This then will be our answer to the question just asked.

We begin with two standard expressions for the de Sitter metric, the static form 


$$
d s^{2}=\frac{3}{\lambda}\left(-\left(1-R^{2}\right) d T^{2}+\frac{d R^{2}}{1-R^{2}}+R^{2} d \Omega^{2}\right) \quad R<1
$$

and the Kantowski-Sachs form

$$
d s^{2}=\frac{3}{\lambda}\left(-\frac{d T^{2}}{T^{2}-1}+\left(T^{2}-1\right) d R^{2}+T^{2} d \Omega^{2}\right) \quad T>1
$$

where in both cases $d \Omega^{2}$ is the metric on $\mathbf{S}^{2}$. Neither metric covers the entire spacetime, but they are locally de Sitter and this is all we need for our present purposes.

The static form of the metric can be taken to the conformal gauge by means of a rescaling of $R$ :

$$
\begin{aligned}
& \tau=T \quad \tanh \rho=R \quad \Rightarrow \\
& d s^{2}=\frac{3}{\lambda}\left(\left(1-\tanh ^{2} \rho\right)\left(-d \tau^{2}+d \rho^{2}\right)+\tanh ^{2} \rho d \Omega^{2}\right) .
\end{aligned}
$$

Define

$$
U=\tau-\rho \quad V=\tau+\rho
$$

and perform the reparametrization

$$
U=U(u) \quad V=V(v) .
$$

The metric then takes the form

$$
d s^{2}=\frac{3}{\lambda}\left(-\left(1-\tanh ^{2} F\right) \frac{\partial U}{\partial u} \frac{\partial V}{\partial v} d u d v+\tanh ^{2} F d \Omega^{2}\right)
$$

where

$$
F=\frac{1}{2}(V(v)-U(u)) .
$$

But this means that $F$ is an arbitrary solution of the two dimensional wave equation. Transforming to a new set of coordinates

$$
u=t-r \quad v=t+r
$$


the metric takes the form

$$
d s^{2}=\frac{3}{\lambda}\left(\left(1-\tanh ^{2} F\right)\left(F^{2}-\dot{F}^{2}\right)\left(-d t^{2}+d r^{2}\right)+\tanh ^{2} F d \Omega^{2}\right),
$$

where $F$ is an arbitrary solution of the two dimensional wave equation in the coordinates $r$ and $t$, a prime denotes differentiation with respect to $r$ and a dot with respect to $t$. This is the form of the metric that we were after. Treating the Kantowski-Sachs metric in the same way we obtain

$$
d s^{2}=\frac{3}{\lambda}\left(\left(\operatorname{coth}^{2} F-1\right)\left(\dot{F}^{2}-F^{2}\right)\left(-d t^{2}+d r^{2}\right)+\operatorname{coth}^{2} F d \Omega^{2}\right) .
$$

For our purposes this is all we need.

To embed a locally spherically symmetric three-space with the metric (2) all that is needed is to choose initial data for the two dimensional wave equation. The initial values of $F$ and $\dot{F}$ at any fixed value of $t=t_{0}$ are at our disposal, so if $|a f|>\sqrt{3 / \lambda}$ we set

$$
\begin{gathered}
g_{\theta \theta}=\frac{3}{\lambda} \operatorname{coth}^{2} F=a^{2} f^{2} \\
g_{r r}=\frac{3}{\lambda}\left(\operatorname{coth}^{2} F-1\right)\left(\dot{F}^{2}-F^{2}\right)=a^{2} .
\end{gathered}
$$

and we are done: The spatial metric at $t=t_{0}$ is the locally spherically symmetric metric (2), and the initial values determine a solution $F(r, t)$ of the wave equation that yields the locally de Sitter metric (13). There are no additional restrictions on the function $f$ in this case.

The story is different if $|a f|<\sqrt{3 / \lambda}$. This time we set

$$
\begin{gathered}
g_{\theta \theta}=\frac{3}{\lambda} \tanh ^{2} F=a^{2} f^{2} \\
g_{r r}=\frac{3}{\lambda}\left(1-\tanh ^{2} F\right)\left(F^{\prime 2}-\dot{F}^{2}\right)=a^{2} .
\end{gathered}
$$

Now there is an additional restriction since $r$ will be a spatial coordinate only if 


$$
g_{r r}>0 \Rightarrow F^{2}-\dot{F}^{2}>0 .
$$

Hence $\dot{F}$ cannot be chosen quite arbitrarily. Moreover from the relation between $f$ and $F$ it follows that

$$
f^{\prime}=0 \quad \Rightarrow \quad F^{\prime}=0 \text {. }
$$

Eqs. (18) and (19) imply that we cannot allow the function $f$ in the threemetric (2) to attain a minimum or a maximum at a radius smaller than $\sqrt{3 / \lambda}$. In particular only handles whose minimum radius exceeds $\sqrt{3 / \lambda}$ can occur. This completes our rederivation of the central result of ref. [1].

The reader may worry that $|a f|$ is a function, and it may be larger or smaller than $\sqrt{3 / \lambda}$ depending on its argument. Actually there is no problem here, as an explicit example will make clear. Set

$$
F=\frac{1}{2} \ln \frac{r-t}{r+t},
$$

which is a real solution of the wave equation if $r>t$. It is easy to show that

$$
\operatorname{coth} F=-\frac{r}{t}
$$

and that the locally de Sitter metric (13) becomes

$$
d s^{2}=\frac{3}{\lambda} \frac{1}{t^{2}}\left(-d t^{2}+d r^{2}+r^{2} d \Omega^{2}\right),
$$

which represents a portion of de Sitter space foliated by flat spaces. When $r<t$ it happens that the argument of the logarithm becomes negative so that $F$ develops an imaginary part. This is just what is needed in order to turn the cotangens hyperbolicus into tangens hyperbolicus, so that the other form of the metric applies.

Finally we observe that our argument can be varied a little. An alternative form of the intrinsic de Sitter metric is

$$
d s^{2}=\frac{3}{\lambda}\left(-\frac{d T^{2}}{T^{2}+1}+\left(T^{2}+1\right) d R^{2}+T^{2} d \sigma^{2}\right)
$$

where $d \sigma^{2}$ is the metric on $\mathbf{H}^{2}$. Treating this line element in the same way as above we arrive at 


$$
d s^{2}=\frac{3}{\lambda}\left(\left(1+\tan ^{2} F\right)\left(\dot{F}^{2}-F^{2}\right)\left(-d t^{2}+d r^{2}\right)+\tan ^{2} F d \sigma^{2}\right) .
$$

Possible initial data slices now include hyperbolic three-space $\mathbf{H}^{3}$ and the hyperbolic handle $\mathbf{H}^{2} \times \mathbf{E}$.

\section{MODEL GEOMETRIES.}

This section is intended to remind the reader of some well known facts [5]. If (for convenience) we set $\lambda=3$ then de Sitter space can be defined as the hyperboloid

$$
X^{2}+Y^{2}+Z^{2}+U^{2}-V^{2}=1
$$

sitting inside a five dimensional Minkowski space with the metric

$$
d s^{2}=d X^{2}+d Y^{2}+d Z^{2}+d U^{2}-d V^{2} .
$$

The isometry group of de Sitter space is therefore the Lorentz group $S O(4,1)$. The embedding space coordinates are very useful for many calculations. In particular, let us consider various spacelike hypersurfaces in de Sitter space.

Intersecting the hyperboloid with the spacelike hyperplanes

$$
V=\sinh t \quad \Rightarrow \quad X^{2}+Y^{2}+Z^{2}+U^{2}=\cosh ^{2} t
$$

we obtain a foliation of de Sitter space with 3-spheres. These 3-spheres turn out to be totally umbilic (that is to say their first and second fundamental forms are proportional). They are totally geodesic and have unit radius if and only if the spacelike plane goes through the origin. Moving the family of spacelike planes around with isometries it is clear that there is a totally geodesic sphere through any point in de Sitter space.

Intersecting the hyperboloid with the timelike hyperplanes

$$
U=\cosh t
$$

we get a foliation of a region of de Sitter space with totally umbilic hyperbolic 3 -spaces. (Intersecting with a timelike hyperplane through the origin yields 
a totally geodesic three-dimensional de Sitter space.) Intersecting with null hyperplanes

$$
U+V=\frac{1}{t}, \quad t>0
$$

we obtain a foliation of "one half" of de Sitter space with totally umbilic flat 3 -spaces. Intersection with a null plane through the origin yields a totally geodesic null hypersurface. Two null planes in five dimensional Minkowski space intersect in a 3-space (unless the null planes are parallel), and the intersection of this 3-space with the de Sitter hyperboloid is a 2-sphere.

We have seen how three of the model geometries $\left(\mathbf{S}^{3}, \mathbf{H}^{3}\right.$ and $\left.\mathbf{E}^{3}\right)$ can be embedded in de Sitter space. To see that the handle can occur, intersect with the upper branch of the spacelike surface

$$
V^{2}-U^{2}=\sinh ^{2} t \quad \Rightarrow \quad X^{2}+Y^{2}+Z^{2}=\cosh ^{2} t .
$$

This is indeed the handle $\mathbf{S}^{2} \times \mathbf{E}$, with the radius of the 2 -sphere necessarily larger than one. Finally we can intersect the hyperboloid with the surface

$$
Z^{2}+U^{2}=\cosh ^{2} t \quad \Rightarrow \quad X^{2}+Y^{2}-V^{2}=-\sinh ^{2} t .
$$

The intersection has the geometry of $\mathbf{H}^{2} \times \mathbf{S}^{1}$. Now $\mathbf{H}^{2} \times \mathbf{E}$ is also one of Thurston's model geometries. If we can "unwrap" the circle it follows that this model geometry also can occur in a locally de Sitter spacetime. Actually the function $F$ in the metric (24) can be arranged so that this is true but it appears that this spatial geometry cannot be embedded in de Sitter space. We will return to this point in section 6 .

Our last two examples are not umbilic surfaces but they do inherit enough Killing vectors from de Sitter space to be homogeneous spaces, and their second fundamental forms share the symmetries of the induced metric.

\section{VISUALIZING DE SITTER SPACE.}

We would now like to show some pictures of the above. This can be done if we restrict ourselves to $2+1$ dimensions and use the methods of ref. [6], which have proved quite useful to derive various properties of locally anti-de Sitter spaces. Naturally there are features of $3+1$ dimensional spacetimes 
that cannot be seen in the lower dimension, but as far as the properties of locally spherically symmetric spacetimes are concerned the restriction is harmless. Thus we consider the hyperboloid

$$
X^{2}+Y^{2}+U^{2}-V^{2}=1
$$

in ordinary Minkowski space. We use a function of $V$ as an intrinsic time coordinate; at constant $V$ space is a sphere. As map-makers know we cannot make a two dimensional map of a sphere unless we give up manifest spherical symmetry; our choice is to make a stereographic projection onto the unit disk of each hemisphere separately. Then we arrive at the coordinate system $(x, y, t)$, where

$$
\begin{array}{cc}
X=\frac{2 x}{1+r^{2}} \frac{1}{\cos t} & Y=\frac{2 y}{1+r^{2}} \frac{1}{\cos t} \\
U= \pm \frac{1-r^{2}}{1+r^{2}} \frac{1}{\cos t} & V=\tan t,
\end{array}
$$

and we use the upper sign in the Northern hemisphere and the lower sign in the Southern. Note that

$$
r^{2} \equiv x^{2}+y^{2} \leq 1
$$

The metric in these coordinates is

$$
d s^{2}=\frac{1}{\cos ^{2} t}\left(-d t^{2}+\frac{4}{\left(1+r^{2}\right)^{2}}\left(d x^{2}+d y^{2}\right)\right) .
$$

The time coordinate $t$ goes from $-\pi / 2$ to $\pi / 2$, and we can attach a conformal boundary consisting of two spheres at $t= \pm \pi / 2$. We can now draw pictures in coordinate space. Our first picture shows de Sitter space as two beer cans whose cylindrical boundaries are to be identified. The scaling of the radial coordinate was chosen so that the slope of a light ray is a function of $r$ only.

We observe that all timelike and null geodesics acquire a future endpoint on future infinity $i^{+}$and a past endpoint on past infinity $i^{-}$, while all spatial geodesics are closed and have circumference $2 \pi$.

Totally geodesic surfaces are easy to find since they are the intersection of the hyperboloid with hyperplanes through the origin in embedding space. Let us begin with totally geodesic null surfaces which result if the hyperplane 


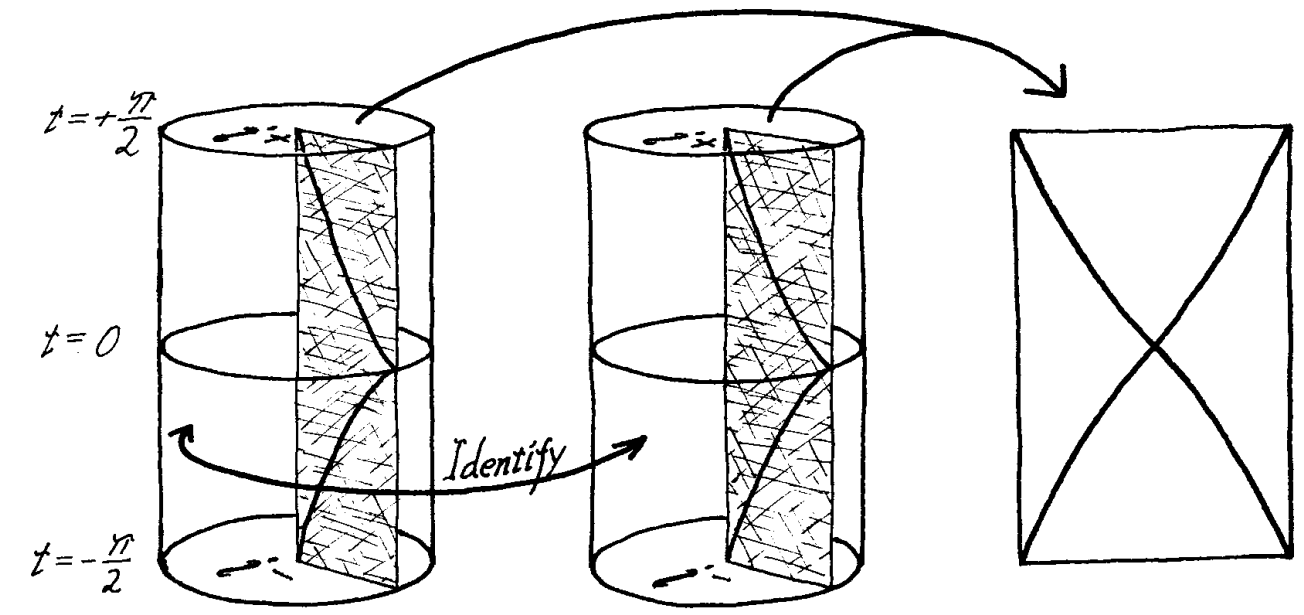

Figure 1: Two beer cans representing de Sitter space. Surfaces of constant $t$ are pairs of disks representing the two hemispheres of a stereographically projected 2 -sphere. The pair of disks on top of the cans represents $i^{+}$. The shaded surface is a Carter-Penrose diagram, each interior point of which represents a circle. The curves on this surface are light rays. Such diagrams are usually drawn so that the slope of a radial light ray is unity but this is not the case here. Our radial coordinate is chosen so that the slope of any light ray depends on $r$ only.

through the origin is chosen to be null. It is easy to check that there is a one-to-one correspondence between such surfaces and the set of lightcones with a vertex on $i^{+}$. (For the twistor theorist this means that $i^{+}$can be thought of as mini-twistor space.) The next picture shows two such surfaces, chosen so that they are rotationally symmetric in our picture.

The isometry group of $2+1$ dimensional de Sitter space is the Lorentz group $S O(3,1)$, which acts like the group of Möbius transformations on $i^{+}$. In particular a Lorentz boost $J_{U V}$ has a bifurcate Killing horizon that coincides with the light cones with vertices at $r=0$; the flow is timelike inside the lightcones and spacelike outside. By symmetry all light cones with vertices on $i^{+}$are Killing horizons. Two important properties follow immediately: 

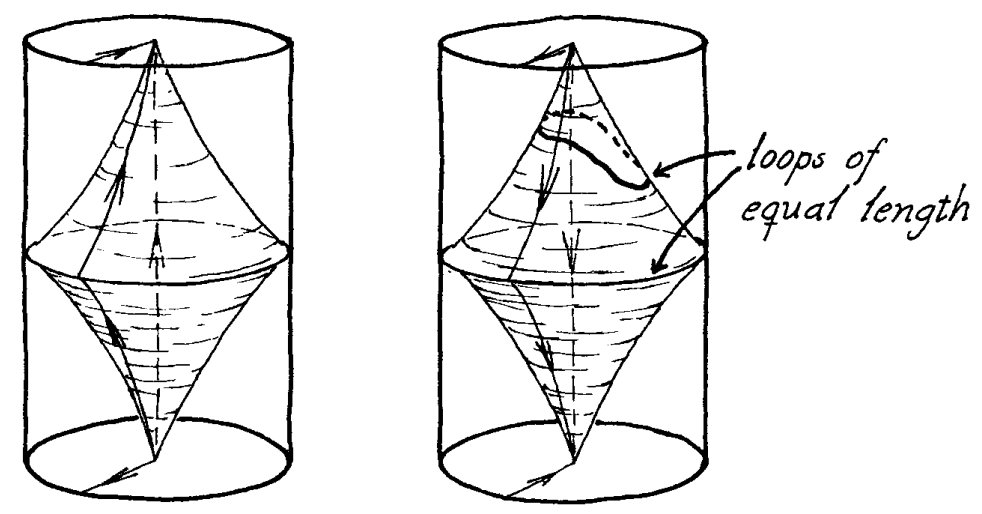

Figure 2: A lightcone with its vertex on $i^{+}$is always totally geodesic. A pair of such lightcones make up a bifurcate Killing horizon; the flow of the relevant Killing vector $J_{U V}$ is indicated by the arrows. Because the surface is a Killing horizon all spacelike loops going around it have equal length. This helps to give a feeling for how distances are distorted by the picture.

First all spacelike loops going around such a cone have equal length (equal to $2 \pi$ with our choice of $\lambda$ ). As a consequence the intersection of two light cones with vertices on $i^{+}$is always a circle with circumference $2 \pi$.

Lorentz boosts correspond to hyperbolic Möbius transformations on $i^{+}$ while rotations correspond to elliptic Möbius transformations; rotations have two timelike lines of fixed points which can be made into world lines of point particles, if this is wanted [7]. A general Lorentz transformation can be written as a combination of a boost and a rotation and is called a four screw. It has no fixed point inside de Sitter space but two fixed points on $i^{+}$, where it gives what one calls a loxodromic Möbius transformation. Finally a null boost has a single fixed point on $i^{+}$and a single light cone for its (degenerate) Killing horizon; there are two lightlike lines of fixed points going through de Sitter space.

We may note that if we identify points along the Killing flow lines of a boost (say $J_{U V}$ for definiteness) we obtain - in the regions where the flow is spacelike - the spatial topology of a torus. This is the de Sitter analogue of Misner space; the anti-de Sitter analogue is the BTZ black hole [6]. 
Let us now consider foliations by spacelike surfaces. In particular we want to draw pictures of the two dimensional analogues $\mathbf{S}^{2}, \mathbf{E}^{2}$ and $\mathbf{S}^{1} \times \mathbf{E}$ of the three dimensional model geometries discussed in section 3. Note that the distinct three dimensional possibilities $\mathbf{H}^{2} \times \mathbf{S}^{1}$ and $\mathbf{S}^{2} \times \mathbf{E}$ collapse to one example only in $2+1$ dimensions. We begin with spheres. A foliation with surfaces of constant $t$ consists of contracting and expanding totally umbilic 2 -spheres; only the pair of disks at $t=0$ represents a totally geodesic sphere. Moving this exceptional sphere around with suitable isometries will give a foliation with totally geodesic spheres of the interior of a pair of totally geodesic null surfaces.
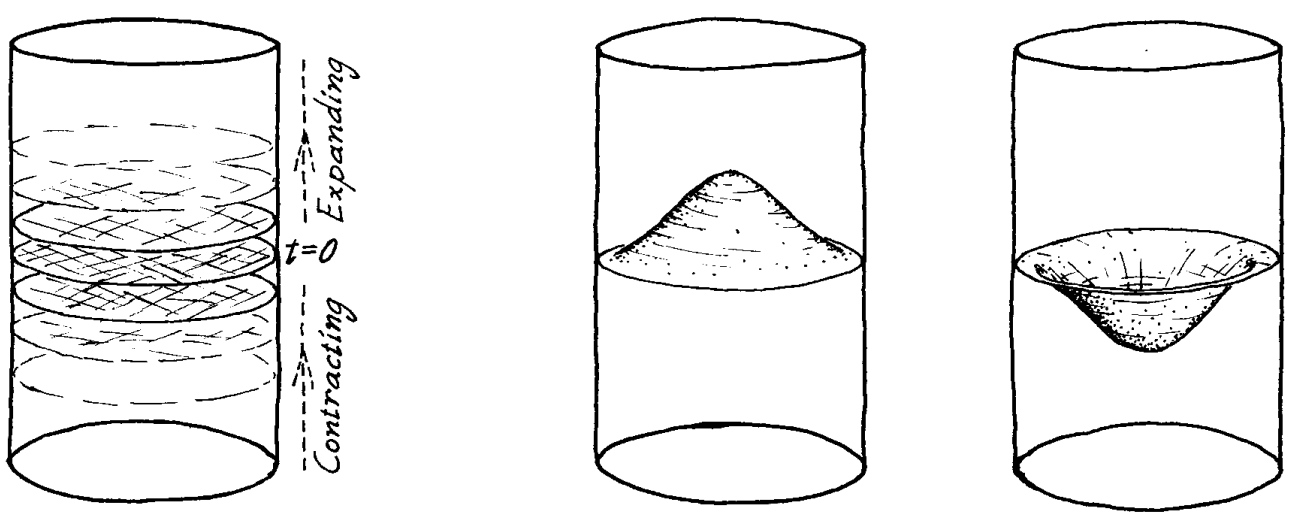

Figure 3: On the left de Sitter space is shown foliated by expanding and contracting spheres; only the exceptional sphere at $t=0$ is totally geodesic (only one can is shown). To the right the totally geodesic sphere has been moved to another position by means of an isometry.

We will take special interest in surfaces that behave asymptotically like one of the model geometries $\mathbf{H}^{2}, \mathbf{E}^{2}$ and $\mathbf{S}^{1} \times \mathbf{E}$. This means that they have to intersect $i^{+}$in a circle, a point or two points, respectively (Figure 4). As we saw in section 3, intersecting the de Sitter hyperboloid with a family of null planes gives a foliation in terms of flat surfaces, while intersecting with timelike planes sufficiently far from the origin gives a foliation with expanding hyperbolic planes. Following section 3 we can also find a foliation with cylinders; we draw this in two different ways to remind the reader that 


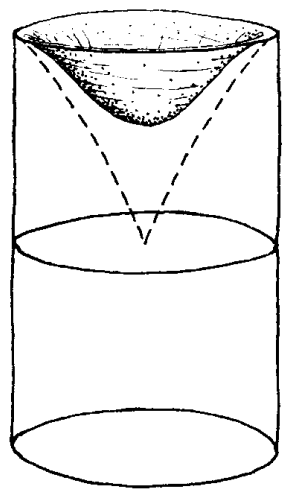

(a)

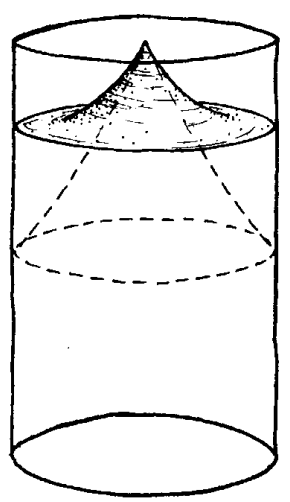

(c)
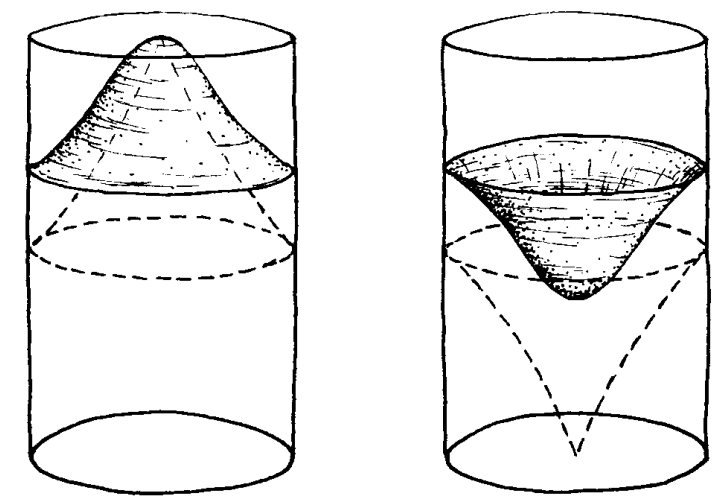

(b)
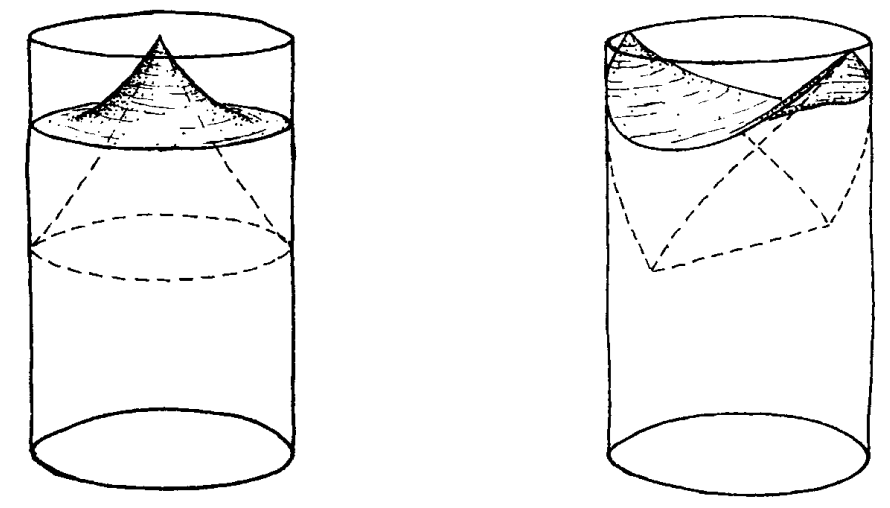

(d)

Figure 4: Other model geometries, with their Cauchy horizons shown dashed: (a) A hyperbolic plane $\mathbf{H}^{2}$ (only one can is shown - the other is empty). (b) A flat space $\mathbf{E}^{2}$. (c) A cylinder $\mathbf{S}^{1} \times \mathbf{E}$, placed so that its symmetry is manifest in the picture. (d) The same cylinder moved to a new position by an isometry (only one can is shown - the other is identical). 
if a given spatial geometry is displaced by an isometry then its appearance in our pictures will change. In all three cases the foliation covers only a region of de Sitter space. The boundary of this region is the Cauchy horizon, which is a null surface. In the case of the handle the Cauchy horizon grows from a spacelike geodesic which is a circle and has circumference $2 \pi$.

\section{SPACES WITH ASYMPTOTIC REGIONS.}

Given the pictures of the various model geometries it is straightforward to draw their connected sums. In fact we do not have to insist that the surfaces be everywhere locally spherically symmetric. We can wiggle them this way and that. As an example, let us (with Morrow-Jones and Witt [㑑]) consider the problem of attaching asymptotic regions to some spatial slice. Then the thing to remember is that a hyperbolic plane always intersects $i^{+}$in a circle, while flat planes and handles intersect $i^{+}$in respectively one and two points. In our picture what we have to do is to deform the sphere so that it touches $i^{+}$in the "right" way (Figure 5).
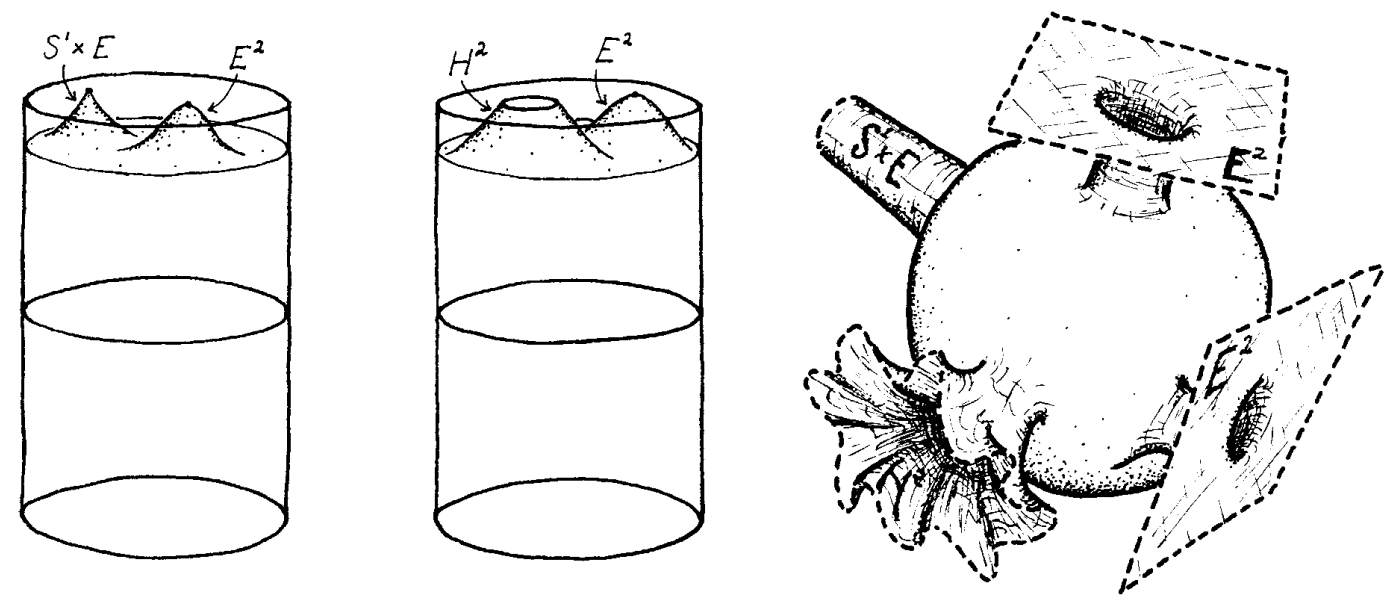

Figure 5: A two-sphere with a number of asymptotic regions attached. Two of the asymptotic regions meet $i^{+}$in such a way that they are flat, one is a cylinder, and one a hyperbolic plane (and the latter contains so much space that it is difficult to draw it in ordinary 3-space). 
It is interesting to ask for the minimum size of the disk that must be removed from the sphere when an asymptotic region is attached. Consider a flat asymptotic region, say. Then we must certainly remove that portion of the sphere that lies in the causal past of the point where the flat space touches $i^{+}$. This is a disk whose circumference is a loop surrounding a light cone with its vertex on $i^{+}$, and as we have seen it necessarily has circumference $2 \pi$. But we can place the asymptotic region as close to this light cone as we want, and therefore the circumference of the disk that we must remove is bounded from below by $2 \pi$. We get the same bound if the asymptotic region is a cylinder or a hyperbolic plane.

Among the 3-spaces considered by Morrow-Jones and Witt [1] is a set of three asymptotically flat spaces connected by two handles. They make the claim that although this space will (given a suitable form of its extrinsic curvature) evolve to a locally de Sitter spacetime, the universal cover of the domain of development cannot be embedded in de Sitter space. Actually this is incorrect.

The argument [1] goes as follows: In four spacetime dimensions the space is simply connected so that we do not have to discuss whether some quotient of de Sitter space will serve as embedding space - it must be de Sitter space itself. This is indeed so. Morrow-Jones and Witt next observe that two flat spaces in de Sitter space necessarily intersect in a sphere (unless they occur at different "times"). This is also correct - in embedding space it is the statement that two non-parallel null planes will intersect in a 3-space, and this 3-space intersects the hyperboloid in a sphere. Finally it is concluded from the picture of the spatial slice that two of the flat regions do not so intersect - but this is not valid since in fact all the spatial planes do intersect, although the two at the ends do so (as it were) within the disks that were removed in attaching the handles that connect them to the plane in the middle.

If this sounds obscure, we hope that it is made clear by a glance at our two beer cans, embellished with a spatial surface carrying precisely the geometry considered by Morrow-Jones and Witt (Figure 6). Although the picture is restricted to $2+1$ dimensional de Sitter space the restriction is irrelevant here. Moreover, in drawing this picture we have in effect solved for the time evolution of these initial data. By the way, the restriction on the minimum radius of a connecting handle (given in ref. [1] and in section 2) also follows from an argument that parallels the argument about the minimum size of 

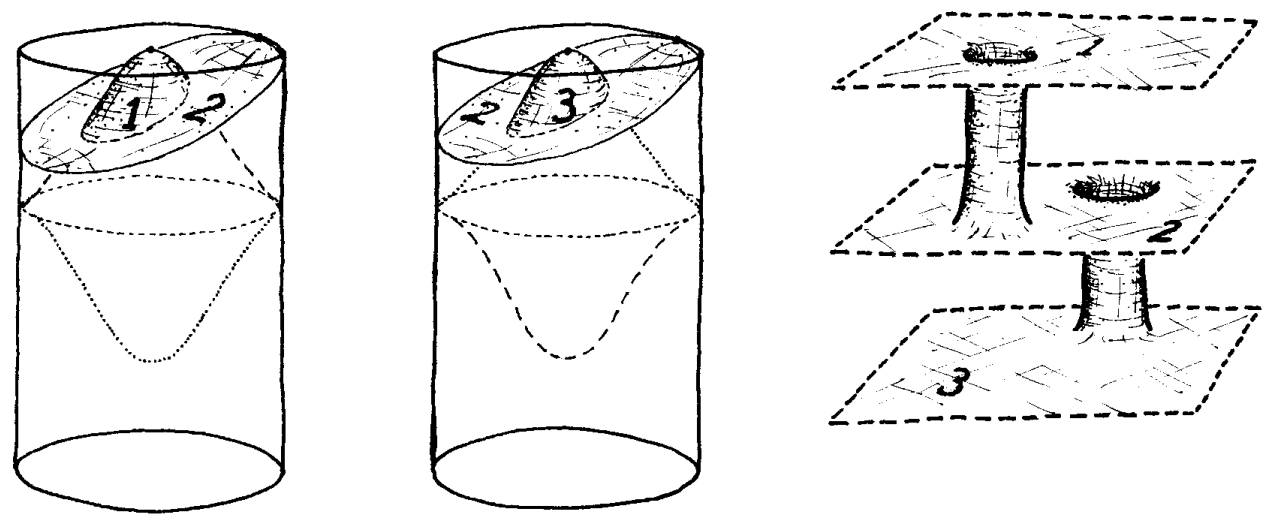

Figure 6: A spatial slice consisting of three flat spaces connected by two handles, and its embedding in de Sitter space. Note that had the intermediate plane 2 not been there then the planes 1 and 3 would have been continued downwards in the cans as indicated by the dashed and dotted lines, respectively. As a result they would have intersected in a circle. This behaviour could not have been guessed just by looking at the schematic picture on the right.

the disk that must be removed when attaching an asymptotic region from the sphere.

Nevertheless there are locally de Sitter spacetimes whose universal covers cannot be regarded as subsets of de Sitter space; we devote section 6 to this topic.

\section{WHY DE SITTER SPACE IS NOT ENOUGH.}

A relativistic space form, by definition, is a complete Lorentzian manifold of constant curvature. They have been completely classified [8]. The universal cover of a relativistic space form is de Sitter space, anti-de Sitter space or Minkowski space depending on the curvature. Our definition of a spacetime was made in the spirit of canonical gravity and does not require completeness. All that we require is the domain of development of some smooth initial data surface. The classification of such spacetimes is a much harder problem and- 
at least to our knowledge - it has been achieved only in $2+1$ dimensions [3] In this connection it was pointed out by Mess [3] that there are smooth locally de Sitter domains of development (complete to the future) whose universal covers cannot be embedded in de Sitter space.

So how can we obtain a smooth surface carrying locally de Sitter data that cannot be embedded in de Sitter space? This is not so difficult. Suppose that we remove a pair of antipodal points from a sphere so that its topology becomes that of a cylinder, and then go to its universal covering space. A similar manœuvre carried through for $2+1$ dimensional de Sitter space entails removing two antipodal timelike lines and going to the universal covering space of the remaining (incomplete) spacetime. To draw the picture it is convenient to place the timelike lines to be removed on the boundary of the cans. The universal covering space then consists of an infinite set of cans with lines cut out of the boundary; the sides of the cans are to be identified pairwise in an obvious manner (Figure 7). Now consider a particular can and insert a "handle" (actually half a handle) that touches what used to be $i^{+}$in precisely the two points that we have removed from the top. Draw a similar surface in all the other cans. This is a smooth spacelike surface of topology $\mathbf{R}^{2}$ - in effect, we have "unwrapped" the cylinder. Its intrinsic geometry is flat but unlike the family of embedded flat spaces considered earlier it is not an umbilic surface. Moreover its Cauchy development is smooth and locally de Sitter by construction, and it is complete to the future. If we try to add a conformal boundary $i^{+}$we run into problems, but then a smooth conformal completion of the domain of development was never promised. In its past there is a Cauchy horizon that grows from a non-closed spacelike geodesic of infinite length - which is one way to see that this spacetime definitely cannot be embedded in de Sitter space since the latter does not have such geodesics. Hence this is the example that we were after.

Let us mention in passing that if we start from the de Sitter analogue of Misner space (as briefly described in section 4) and then "cut out a wedge" bounded by timelike surfaces - that is, if we identify points along the flow lines of the Killing vector $J_{X Y}$ - then the spatial topology is again a torus, but a torus whose covering space (in the generic case) is the spacetime that we have just discussed.

The example just given works specifically in $2+1$ dimensions, but it is not difficult to modify it so that it works in $3+1$ dimensions. We start out by considering the model geometry $\mathbf{H}^{2} \times \mathbf{S}^{1}$ embedded as a hypersurface in 


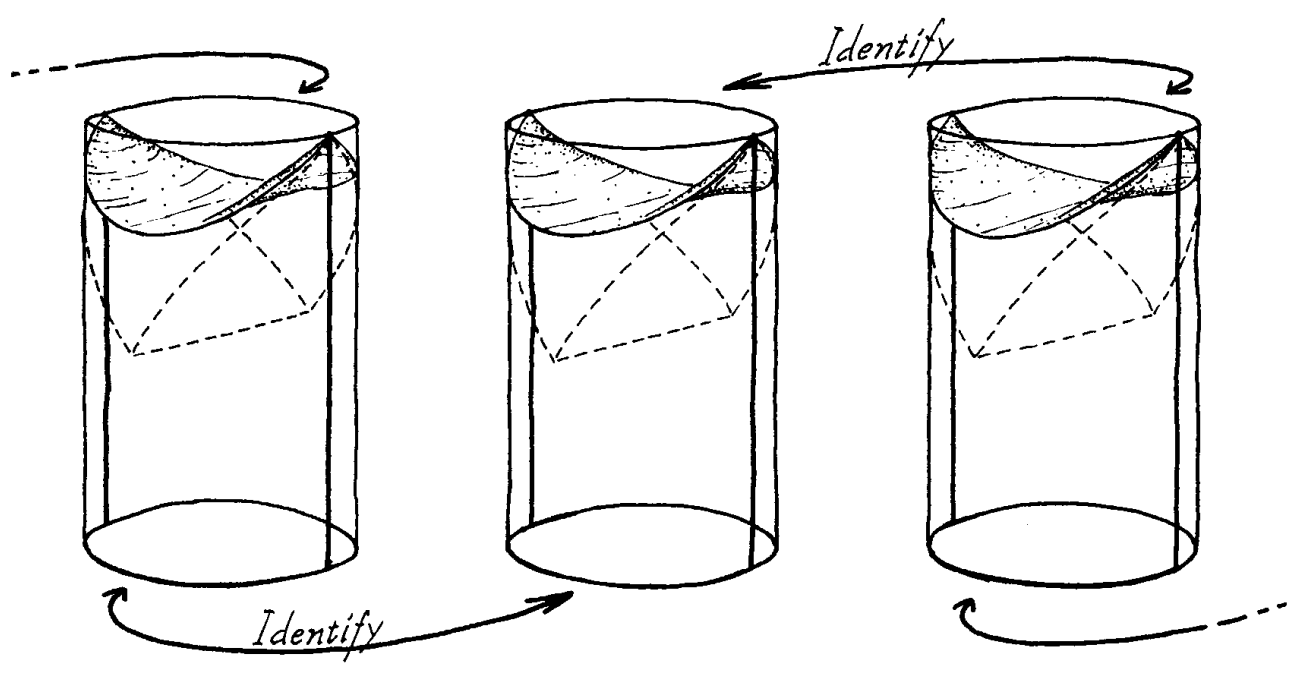

Figure 7: A smooth locally de Sitter and future complete domain of development; in effect an "unrolled" handle. Now there is an infinite set of cans (only three cans are drawn) with sides identified as shown. This is possible because a pair of timelike geodesics on each boundary has been removed. The domain of development of the embedded surface is simply connected, yet it cannot be embedded in de Sitter space. Note that the timelike geodesics that we have removed lie outside the Cauchy development (indicated with dashed lines) of the surface.

de Sitter space through

$$
Y^{2}+U^{2}=\cosh ^{2} \tau \quad \Leftrightarrow \quad X^{2}+Z^{2}-V^{2}=-\sinh ^{2} \tau .
$$

Our aim is to "unwrap" the circle. The Cauchy development of the embedded hypersurface is the future of the spatial geodesic

$$
X=Z=V=0 .
$$

The circle becomes unwrapped if we are able to unwrap the flow lines of the Killing vector $J_{Y U}$ (that generates rotations). To succeed in this we must remove the fixed points of this Killing vector from de Sitter space; in equations the fixed points are given by 


$$
J_{Y U}=Y \partial_{U}-U \partial_{Y}=0 \quad \Rightarrow \quad Y=U=0 .
$$

Metrically this is a $1+1$ dimensional de Sitter space; for us it is important to observe that it has zero intersection with the Cauchy development of the embedded hypersurface. On the other hand the fixed point set and the embedded hypersurface touch $i^{+}$in the same circle. If we think of de Sitter space as a set of foliating 3-spheres we see that the fixed point set gives a circle in each 3 -sphere - and a circle is precisely what we must remove in order to make a 3-sphere multiply connected. Therefore once the fixed point set has been removed we have an incomplete, multiply connected spacetime and we can go to its universal covering space. Having done this we have an embedding of Thurston's model geometry $\mathbf{H}^{2} \times \mathbf{E}$ in an incomplete spacetime that is "larger" than de Sitter space; nevertheless the Cauchy development of our model geometry is not only simply connected but also complete to the future. This Cauchy development is in itself a spacetime in our sense.

To visualize this construction one can draw a series of equal- $t$ slices of $3+1$ dimensional de Sitter space, that is a series of stereographically projected 3spheres, and study how the hypersurface $\mathbf{H}^{2} \times \mathbf{S}^{1}$ intersects these slices. In effect all that one has to do is to take equal- $t$ slices of fig. 7 and rotate them around a suitable axis. The details are left to the interested reader.

We have embedded the model geometry $\mathbf{H}^{2} \times \mathbf{E}$ in a locally de Sitter spacetime which cannot itself be found as a subset of de Sitter space. To see that there is no other way to embed this model geometry in de Sitter space we may use eq. (24) to prove that its extrinsic geometry is determined by the requirement that it is embedded in a locally de Sitter space. Therefore its Cauchy development has to be precisely the spacetime that we just constructed, and this spacetime cannot be a subset of de Sitter space since-like its $2+1$ dimensional counterpart - it grows from an infinitely long non-closed spacelike geodesic.

\section{CONCLUSIONS.}

Our conclusions can be summarized like this:

One can write the de Sitter metric in a form that allows one to choose any allowed locally spherically symmetric geometry on a spacelike slice by 
choosing initial data for the two dimensional wave equation.

A similar form exists for spatial geometries of the form (hyperbolic plane) $\times$ (something).

Using a pictorial presentation the restriction to local spherical symmetry everywhere can be dropped and the Cauchy development of any spatial slice is easily studied.

The model geometry $\mathbf{H}^{2} \times \mathbf{E}$ provides an explicit example of a smooth locally de Sitter domain of development whose universal cover cannot be embedded in de Sitter space.

On the other hand three flat asymptotic regions connected by two wormholes can be so embedded (pace previous claims).

As a final comment we observe that de Sitter space is very different from the other two relativistic space forms, Minkowski space and anti-de Sitter space. In de Sitter space asymptotic regions of spacelike slices occur where the slices approach future infinity. Hence the issue of connecting different asymptotic regions with causal curves [9] does not even arise. A little thought will also convince the reader that our examples of simply connected locally de Sitter spacetimes that cannot be embedded in de Sitter space came about precisely because the conformal boundary (or more accurately the would be conformal boundary) is a spacelike surface. Hence this behaviour cannot occur for locally flat or anti-de Sitter spacetimes - a fact which is perfectly well known [3], but perhaps we have managed to convey some extra feeling for why it is true.

Acknowledgements: We thank Dieter Brill for suggesting this problem, and Stefan Åminneborg and Greg Galloway for discussions and help. IB was supported by the NFR.

\section{References}

[1] J. Morrow-Jones and D.M. Witt, Phys. Rev. D48 (1993) 2516. 
[2] W.P. Thurston, Bull. AMS 6 (1982) 357.

[3] G. Mess, Lorentz Spacetimes of Constant Curvature, IHES preprint, April 1990.

[4] A. Ishibashi, T. Koike, M. Siino and S. Kojima, Phys. Rev. D54 (1996) 7303.

[5] E. Schrödinger: Expanding Universes, Cambridge U.P 1956.

[6] S. Åminneborg, I. Bengtsson, S. Holst and P. Peldán, Class. Quant. Grav.

[7] S. Deser and R. Jackiw, Ann. Phys. (NY) 153 (1984) 405.

[8] E. Calabi and L. Markus, Ann. Math. 75 (1962) 63.

[9] J.L. Friedman, K. Schleich and D.M. Witt, Phys. Rev. Lett. 71 (1993) 1486. 